\title{
Super-Hamiltonian Structures and Conservation Laws of a New Six-Component Super-Ablowitz-Kaup-Newell-Segur Hierarchy
}

\author{
Fucai You, ${ }^{1}$ Jiao Zhang, ${ }^{1}$ and Yan Zhao ${ }^{2}$ \\ ${ }^{1}$ Department of Basic Sciences, Shenyang Institute of Engineering, Shenyang 110136, China \\ ${ }^{2}$ College of New Energy, Shenyang Institute of Engineering, Shenyang 110136, China \\ Correspondence should be addressed to Fucai You; fcyou@163.com
}

Received 11 June 2014; Accepted 28 July 2014; Published 20 August 2014

Academic Editor: Huanhe Dong

Copyright (c) 2014 Fucai You et al. This is an open access article distributed under the Creative Commons Attribution License, which permits unrestricted use, distribution, and reproduction in any medium, provided the original work is properly cited.

A six-component super-Ablowitz-Kaup-Newell-Segur (-AKNS) hierarchy is proposed by the zero curvature equation associated with Lie superalgebras. Supertrace identity is used to furnish the super-Hamiltonian structures for the resulting nonlinear superintegrable hierarchy. Furthermore, we derive the infinite conservation laws of the first two nonlinear super-AKNS equations in the hierarchy by utilizing spectral parameter expansions. PACS: 02.30.Ik; 02.30.Jr; 02.20.Sv.

\section{Introduction}

It is well known that many physically important integrable partial differential equations belong to the Ablowitz-KaupNewell-Segur (AKNS) hierarchy [1-3], such as the KdV equation, the $\mathrm{mKdV}$ equation, the nonlinear Schrödinger equation, the Sin-Gordon equation, and the mixed KdVmKdV equation. The AKNS hierarchy is based on the Zakharov and Shabat [4] spectral problem

$$
\left(\begin{array}{l}
\phi_{1} \\
\phi_{2}
\end{array}\right)_{x}=\left(\begin{array}{cc}
-\lambda & q \\
r & \lambda
\end{array}\right)\left(\begin{array}{l}
\phi_{1} \\
\phi_{2}
\end{array}\right) .
$$

It possesses Lax representation, Hamiltonian structures, and infinitely many conserved quantities and can be solved by the method of inverse scattering, Hirota method, Darboux transform, and others.

The superintegrable systems had already aroused strong interest in theoretical physics $[5,6]$, where the fermion fields are added and equally treated with the boson fields. Many classical integrable equations have been extended to the super ones by adding fermion fields, such as the super-AKNS [6-10], the super-KdV [5], the super-Dirac $[9,11,12]$, and the super-Kadomtsev Petviashvili (KP) [13-15]. The superAKNS hierarchy was first proposed in [6] based on the superalgebra $\mathrm{sl}(2, \mathrm{R})$. Extension of this work to other and higher dimensional superalgebras is given in [16]. The superAKNS matrix superspectral problem is

$$
\left(\begin{array}{l}
\psi_{1} \\
\psi_{2} \\
\psi_{3}
\end{array}\right)_{x}=\left(\begin{array}{ccc}
-\lambda & q & \alpha \\
r & \lambda & \beta \\
-\beta & \alpha & 0
\end{array}\right)\left(\begin{array}{l}
\psi_{1} \\
\psi_{2} \\
\psi_{3}
\end{array}\right)
$$

where $\psi_{3}, \alpha$, and $\beta$ are fermion fields. It reduces to the spectral AKNS's system as $\alpha=\beta=0$.

In this paper, we consider a new $3 \times 3$ matrix superspectral problem which generates a six-component super-AKNS hierarchy. As we will show this spectral problem takes the spectral AKNS's system and super-AKNS's system as special cases.

The paper is organized as follows. In Section 2, we will construct a six-component super-AKNS hierarchy related to the $3 \times 3$ matrix superspectral problem. In Section 3, we present the super-Hamiltonian structures for the sixcomponent super-AKNS hierarchy with the help of the supertrace identity. In Section 4, we consider some special reductions of the superintegrable hierarchy. In Section 5, we derive the infinite conservation laws for the associated hierarchy. The last section contains concluding remarks. 


\section{A Six-Component Super-AKNS Hierarchy}

In this section, we will derive a new six-component superAKNS hierarchy associated with the $3 \times 3$ matrix spectral problem. Let $\mathscr{G}$ be a commutative superalgebra over $\mathbb{R}$ and $\widetilde{\mathscr{G}}$ a matrix loop superalgebra over $\mathscr{G}$ with the nondegenerate Killing form. We take a matrix superspectral problem

$$
\begin{array}{r}
\phi_{x}=U \phi=U(u, \lambda) \phi, \quad \phi=\left(\begin{array}{l}
\phi_{1} \\
\phi_{2} \\
\phi_{3}
\end{array}\right), \\
U=\left(\begin{array}{ccc}
-\lambda & q & \alpha_{1} \\
r & \lambda & \beta_{1} \\
\beta_{2} & \alpha_{2} & 0
\end{array}\right),
\end{array}
$$

where $\phi_{x}$ and $\phi_{t}$ denote the partial derivatives with respect to $x$ and $t, u=\left(q, r, \alpha_{1}, \alpha_{2}, \beta_{1}, \beta_{2}\right)^{T} \in \mathscr{G}^{6}$ is a potential consisting of commuting and anticommuting variables, $q, r, \lambda, \phi_{1}, \phi_{2}$ are the commuting variables, which can be indicated by the degree $p$ as $p(q)=p(r)=p(\lambda)=p\left(\phi_{1}\right)=p\left(\phi_{2}\right)=0$, and $\alpha_{1}, \alpha_{2}, \beta_{1}, \beta_{2}, \phi_{3}$ are the anticommuting variables, which can be indicated by the degree $p$ as $p\left(\alpha_{1}\right)=p\left(\alpha_{2}\right)=p\left(\beta_{1}\right)=$ $p\left(\beta_{2}\right)=p\left(\phi_{3}\right)=1$. Here $\lambda$ is assumed to be a constant spectral parameter (i.e., $\lambda_{t}=0$ ).

Let us find the following temporal evolution equation associated with (3):

$$
\phi_{t}=V \phi, \quad V=\left(\begin{array}{ccc}
\frac{A+G}{2} & B & \rho \\
C & \frac{-A+G}{2} & \delta \\
\varepsilon & \tau & G
\end{array}\right) \text {, }
$$

where $A, B, C$, and $G$ are commuting fields and $\rho, \delta, \varepsilon$, and $\tau$ are anticommuting fields. From the stationary zero curvature equation

$$
V_{x}=[U, V],
$$

it gives rise to

$$
\begin{gathered}
A_{x}=2 q C-2 r B+\alpha_{1} \varepsilon-\beta_{1} \tau-\alpha_{2} \delta+\beta_{2} \rho, \\
B_{x}=-2 \lambda B-q A+\alpha_{1} \tau+\alpha_{2} \rho, \\
C_{x}=2 \lambda C+r A+\beta_{1} \varepsilon+\beta_{2} \delta, \\
G_{x}=\alpha_{1} \varepsilon+\beta_{1} \tau+\alpha_{2} \delta+\beta_{2} \rho, \\
\rho_{x}=-\lambda \rho+q \delta-\frac{1}{2} \alpha_{1} A+\frac{1}{2} \alpha_{1} G-\beta_{1} B, \\
\delta_{x}=\lambda \delta+r \rho-\alpha_{1} C+\frac{1}{2} \beta_{1} A+\frac{1}{2} \beta_{1} G, \\
\tau_{x}=-\lambda \tau-q \varepsilon-\frac{1}{2} \alpha_{2} A-\frac{1}{2} \alpha_{2} G+\beta_{2} B, \\
\varepsilon_{x}=\varepsilon-r \tau+\alpha_{2} C+\frac{1}{2} \beta_{2} A-\frac{1}{2} \beta_{2} G .
\end{gathered}
$$

We put $A, B, C, G, \rho, \delta, \varepsilon$, and $\tau$ to be polynomial of $\lambda$ :

$$
A=\sum_{m \geq 0}^{\infty} A_{m} \lambda^{-m}, \quad B=\sum_{m \geq 0}^{\infty} B_{m} \lambda^{-m},
$$

$$
\begin{array}{rlrl}
C & =\sum_{m \geq 0}^{\infty} C_{m} \lambda^{-m}, & G=\sum_{m \geq 0}^{\infty} G_{m} \lambda^{-m}, \\
\rho=\sum_{m \geq 0}^{\infty} \rho_{m} \lambda^{-m}, & \varepsilon=\sum_{m \geq 0}^{\infty} \varepsilon_{m} \lambda^{-m}, \\
\delta=\sum_{m \geq 0}^{\infty} \delta_{m} \lambda^{-m}, & \tau=\sum_{m \geq 0}^{\infty} \tau_{m} \lambda^{-m},
\end{array}
$$

and substituting (7) into (6) and equating the coefficients of $\lambda$, we obtain

$$
\begin{gathered}
A_{m, x}=2 q C_{m}-2 r B_{m}+\alpha_{1} \varepsilon_{m}-\beta_{1} \tau_{m}-\alpha_{2} \delta_{m}+\beta_{2} \rho_{m}, \\
B_{m, x}=-2 B_{m+1}-q A_{m}+\alpha_{1} \tau_{m}+\alpha_{2} \rho_{m}, \\
C_{m, x}=2 C_{m+1}+r A_{m}+\beta_{1} \varepsilon_{m}+\beta_{2} \delta_{m}, \\
G_{m, x}=\alpha_{1} \varepsilon_{m}+\beta_{1} \tau_{m}+\alpha_{2} \delta_{m}+\beta_{2} \rho_{m}, \\
\rho_{m, x}=-\rho_{m+1}+q \delta_{m}-\frac{1}{2} \alpha_{1} A_{m}+\frac{1}{2} \alpha_{1} G_{m}-\beta_{1} B_{m}, \\
\delta_{m, x}=\delta_{m+1}+r \rho_{m}-\alpha_{1} C_{m}+\frac{1}{2} \beta_{1} A_{m}+\frac{1}{2} \beta_{1} G_{m}, \\
\tau_{m, x}=-\tau_{m+1}-q \varepsilon_{m}-\frac{1}{2} \alpha_{2} A_{m}-\frac{1}{2} \alpha_{2} G_{m}+\beta_{2} B_{m}, \\
\varepsilon_{m, x}=\varepsilon_{m+1}-r \tau_{m}+\alpha_{2} C_{m}+\frac{1}{2} \beta_{2} A_{m}-\frac{1}{2} \beta_{2} G_{m} .
\end{gathered}
$$

Upon choosing the initial data

$$
\begin{gathered}
A_{0}=-2, \quad B_{0}=C_{0}=\rho_{0}=\delta_{0}=\varepsilon_{0}=\tau_{0}=0, \\
G_{0}=-g_{0}=\text { constant }
\end{gathered}
$$

then the recursion relations in (8) uniquely define a series of sets of differential polynomial functions in $u$ with respect to $x$. The first two sets are as follows:

$$
\begin{gathered}
B_{1}=q, \quad C_{1}=r, \quad A_{1}=0, \\
G_{1}=0, \quad \rho_{1}=-\frac{1}{2} \alpha_{1}\left(g_{0}-2\right), \quad \tau_{1}=\frac{1}{2} \alpha_{2}\left(g_{0}+2\right), \\
\delta_{1}=\frac{1}{2} \beta_{1}\left(g_{0}+2\right), \quad \varepsilon_{1}=-\frac{1}{2} \beta_{2}\left(g_{0}-2\right) \\
B_{2}=-\frac{1}{2}\left(q_{x}-g_{0} \alpha_{1} \alpha_{2}\right), \quad C_{2}=\frac{1}{2}\left(r_{x}+g_{0} \beta_{1} \beta_{2}\right), \\
\rho_{2}=-\alpha_{1, x}+\frac{1}{2} g_{0}\left(\alpha_{1, x}+q \beta_{1}\right) \\
\tau_{2}=-\alpha_{2, x}-\frac{1}{2} g_{0}\left(\alpha_{2, x}-q \beta_{2}\right)
\end{gathered}
$$




$$
\begin{gathered}
\delta_{2}=\beta_{1, x}+\frac{1}{2} g_{0}\left(\beta_{1, x}+r \alpha_{1}\right), \\
A_{2}=q r+\alpha_{1} \beta_{2}-\alpha_{2} \beta_{1}-\frac{1}{2} g_{0}\left(\alpha_{1} \beta_{2}+\alpha_{2} \beta_{1}\right), \\
\varepsilon_{2}=\beta_{2, x}-\frac{1}{2} g_{0}\left(\beta_{2, x}-r \alpha_{2}\right), \\
G_{2}=\alpha_{1} \beta_{2}+\alpha_{2} \beta_{1}-\frac{1}{2} g_{0}\left(\alpha_{1} \beta_{2}-\alpha_{2} \beta_{1}\right) .
\end{gathered}
$$

From the recursion relations in (8), we can obtain the hereditary recursion operator $L$ which satisfies that

$$
\begin{gathered}
\left(C_{m+1}, B_{m+1},-\varepsilon_{m+1},-\tau_{m+1}, \delta_{m+1}, \rho_{m+1}\right)^{T} \\
=L\left(C_{m}, B_{m},-\varepsilon_{m},-\tau_{m}, \delta_{m}, \rho_{m}\right)^{T}
\end{gathered}
$$

where

$$
\begin{aligned}
& L=\left(\begin{array}{lll}
L_{11} & L_{12} & L_{13} \\
L_{21} & L_{22} & L_{23} \\
L_{31} & L_{32} & L_{33}
\end{array}\right) \\
& L_{11}=\left(\begin{array}{cc}
-\frac{1}{2} \partial+r \partial^{-1} q & -r \partial^{-1} r \\
q \partial^{-1} q-\frac{1}{2} \alpha_{1} & \frac{1}{2} \partial-q \partial^{-1} r
\end{array}\right) \text {, } \\
& L_{12}=\left(\begin{array}{cc}
-\frac{r}{2} \partial^{-1} \alpha_{1} & \frac{r}{2} \partial^{-1} \beta_{1} \\
-\partial^{-1} \alpha_{1} & \partial^{-1} \beta_{1}
\end{array}\right), \\
& L_{13}=\left(\begin{array}{cc}
-\frac{r}{2} \partial^{-1} \alpha_{2} & \frac{r}{2} \partial^{-1} \beta_{2} \\
-\partial^{-1} \alpha_{2} & \partial^{-1} \beta_{2}-\frac{1}{2} \alpha_{2}
\end{array}\right) \text {, } \\
& L_{21}=\left(\begin{array}{cc}
-\alpha_{2}-\beta_{2} \partial^{-1} q & \beta_{2} \partial^{-1} r \\
-\alpha_{2} \partial^{-1} q & \alpha_{2} \partial^{-1} r+\beta_{2}
\end{array}\right) \text {, } \\
& L_{22}=\left(\begin{array}{cc}
-\partial-\partial^{-1} \alpha_{1}-\frac{1}{2} \beta_{2} \partial^{-1} \alpha_{1} & -r-\beta_{2} \partial^{-1} \beta_{1} \\
q+\alpha_{2} \partial^{-1} \alpha_{1} & \partial
\end{array}\right), \\
& L_{23}=\left(\begin{array}{cc}
\beta_{2} \partial^{-1} \alpha_{2} & 0 \\
0 & -\alpha_{2} \partial^{-1} \beta_{2}
\end{array}\right) \text {, } \\
& L_{31}=\left(\begin{array}{cc}
-\alpha_{1}+\beta_{1} \partial^{-1} q & -\beta_{1} \partial^{-1} r \\
\alpha_{1} \partial^{-1} q & -\alpha_{1} \partial^{-1} r+\beta_{1}
\end{array}\right) \\
& L_{32}=\left(\begin{array}{cc}
-\beta_{1} \partial^{-1} \alpha_{1} & 0 \\
0 & \alpha_{1} \partial^{-1} \beta_{1}
\end{array}\right), \\
& L_{33}=\left(\begin{array}{cc}
-\partial & r+\beta_{1} \partial^{-1} \beta_{2} \\
-q-\alpha_{1} \partial^{-1} \alpha_{2} & \partial+\partial^{-1} \beta_{2}-\frac{1}{2} \alpha_{1} \partial^{-1} \beta_{2}
\end{array}\right) \text {. }
\end{aligned}
$$

Taking

$$
V^{(n)}=\left(\lambda^{n} V\right)_{+}=\sum_{j=0}^{n}\left(\begin{array}{ccc}
\frac{A_{j}+G_{j}}{2} & B_{j} & \rho_{j} \\
C_{j} & \frac{-A_{j}+G_{j}}{2} & \delta_{j} \\
\varepsilon_{j} & \tau_{j} & G_{j}
\end{array}\right) \lambda^{n-j},
$$

here $\left(\lambda^{n} V\right)_{+}$denotes the polynomial part of $\lambda^{n} V$. tion)

The compatibility conditions (i.e., zero curvature equa-

$$
U_{t}-V_{x}^{(n)}+\left[U, V^{(n)}\right]=0
$$

of the matrix superspectral problems

$$
\phi_{x}=U \phi, \quad \phi_{t_{n}}=V^{(n)} \phi, \quad n \geq 0,
$$

determine a new six-component super-AKNS integrable soliton hierarchy

$$
u_{t_{n}}=\left(\begin{array}{c}
q \\
r \\
\alpha_{1} \\
\beta_{1} \\
\alpha_{2} \\
\beta_{2}
\end{array}\right)_{t_{n}}=K_{n}(u)=\left(\begin{array}{c}
-2 B_{n+1} \\
2 C_{n+1} \\
-\rho_{n+1} \\
\delta_{n+1} \\
-\tau_{n+1} \\
\varepsilon_{n+1}
\end{array}\right), \quad n \geq 0 .
$$

\section{The Super-Hamiltonian Structures}

In this section, we will establish the super-Hamiltonian structure of the six-component super-AKNS hierarchy by supertrace identity $[9,17]$

$$
\frac{\delta}{\delta u} \int \operatorname{Str}\left(V \frac{\partial U}{\partial \lambda}\right) d x=\lambda^{-\gamma} \frac{\partial}{\partial \lambda} \lambda^{\gamma} \operatorname{Str}\left(V \frac{\partial U}{\partial u}\right),
$$

where the constant $\gamma$ is determined by

$$
\gamma=-\frac{\lambda}{2} \frac{d}{d \lambda} \ln |\operatorname{Str}(V V)|
$$

Through direct calculations, we have

$$
\begin{gathered}
\operatorname{Str}\left(V \frac{\partial U}{\partial \lambda}\right)=-A, \quad \operatorname{Str}\left(V \frac{\partial U}{\partial q}\right)=C, \\
\operatorname{Str}\left(V \frac{\partial U}{\partial r}\right)=B, \quad \operatorname{Str}\left(V \frac{\partial U}{\partial \alpha_{1}}\right)=-\varepsilon, \\
\operatorname{Str}\left(V \frac{\partial U}{\partial \beta_{1}}\right)=-\tau, \quad \operatorname{Str}\left(V \frac{\partial U}{\partial \alpha_{2}}\right)=\delta, \\
\operatorname{Str}\left(V \frac{\partial U}{\partial \beta_{2}}\right)=\rho .
\end{gathered}
$$

Substituting the above results into the supertrace identity (17) yields that

$$
\frac{\delta}{\delta u} \int-A d x=\lambda^{-\gamma} \frac{\partial}{\partial \lambda} \lambda^{\gamma}(C, B,-\varepsilon,-\tau, \delta, \rho)^{T} .
$$


Comparing the coefficients of $\lambda^{-m-2}$ on both sides of (20) gives rise to

$$
\frac{\delta}{\delta u} \int-A_{m+2} d x=(\gamma-m-1)\left(\begin{array}{c}
C_{m+1} \\
B_{m+1} \\
-\varepsilon_{m+1} \\
-\tau_{m+1} \\
\delta_{m+1} \\
\rho_{m+1}
\end{array}\right), \quad m \geq 0
$$

By employing the computing formula (18) on the constant $\gamma$, we obtain $\gamma=0$. Thus we have

$$
\frac{\delta \mathscr{H}_{m}}{\delta u}=\left(\begin{array}{c}
C_{m+1} \\
B_{m+1} \\
-\varepsilon_{m+1} \\
-\tau_{m+1} \\
\delta_{m+1} \\
\rho_{m+1}
\end{array}\right), \quad \mathscr{H}_{m}=\int \frac{2}{m+1} A_{m+1} d x, \quad m \geq 0
$$

It then follows that the superintegrable hierarchy (16) possesses the following super-Hamiltonian form:

$$
u_{t_{n}}=K_{n}(u)=J \frac{\delta \mathscr{H}_{n}}{\delta u}, \quad n \geq 0,
$$

where the super-Hamiltonian operator $J$ is given by

$$
J=\left(\begin{array}{cccccc}
0 & -2 & 0 & 0 & 0 & 0 \\
2 & 0 & 0 & 0 & 0 & 0 \\
0 & 0 & 0 & 0 & 0 & -1 \\
0 & 0 & 0 & 0 & 1 & 0 \\
0 & 0 & 0 & 1 & 0 & 0 \\
0 & 0 & -1 & 0 & 0 & 0
\end{array}\right)
$$

We note that the recursion operator $L$ is an integrodifferential operator, but the generalized superintegrable system (23) is pure differential equations according to [12].

\section{Reductions}

We now consider the possible reductions of our sixcomponent super-AKNS hierarchy.

Assuming $\alpha_{1}=\alpha_{2}=\beta_{1}=\beta_{2}=0$, hierarchy (23) reduces to the classical AKNS hierarchy [1]. Taking $\alpha_{1}=-\alpha_{2}, \beta_{2}=$ $\beta_{1}$, we can have the super-AKNS hierarchy $[6,9,10]$.

When $n=1$ in (23), we obtain the first-order nonlinear superintegrable equations

$$
\begin{aligned}
& q_{t_{1}}=q_{x}-g_{0} \alpha_{1} \alpha_{2}, \\
& r_{t_{1}}=r_{x}+g_{0} \beta_{1} \beta_{2},
\end{aligned}
$$

$$
\begin{aligned}
& \alpha_{1, t_{1}}=\alpha_{1, x}-\frac{1}{2} g_{0} \alpha_{1, x}-\frac{1}{2} g_{0} q \beta_{1}, \\
& \alpha_{2, t_{1}}=\alpha_{2, x}+\frac{1}{2} g_{0} \alpha_{2, x}-\frac{1}{2} g_{0} q \beta_{2}, \\
& \beta_{1, t_{1}}=\beta_{1, x}+\frac{1}{2} g_{0} \beta_{1, x}+\frac{1}{2} g_{0} r \alpha_{1}, \\
& \beta_{2, t_{1}}=\beta_{2, x}-\frac{1}{2} g_{0} \beta_{2, x}+\frac{1}{2} g_{0} r \alpha_{2} .
\end{aligned}
$$

Taking $n=2$ in (23), we can obtain the second-order nonlinear superintegrable equations

$$
\begin{aligned}
& q_{t_{2}}=-\frac{1}{2} q_{x x}+q^{2} r+\left(g_{0}-1\right)\left(\alpha_{1, x} \alpha_{2}-q \alpha_{1} \beta_{2}\right) \\
& -\left(g_{0}+1\right)\left(\alpha_{2, x} \alpha_{1}+q \alpha_{2} \beta_{1}\right), \\
& r_{t_{2}}=\frac{1}{2} r_{x x}-q r^{2}+\left(g_{0}+1\right)\left(\beta_{1, x} \beta_{2}+r \alpha_{2} \beta_{1}\right) \\
& +\left(g_{0}-1\right)\left(r \alpha_{1} \beta_{2}-\beta_{2, x} \beta_{1}\right), \\
& \alpha_{1, t_{2}}=\left(\frac{1}{2} g_{0}-1\right) \alpha_{1, x x}+\frac{1}{2}\left(g_{0}-1\right) \beta_{1} q_{x}-q \beta_{1, x} \\
& +\frac{1}{2}\left(1-g_{0}\right) q r \alpha_{1}-\alpha_{1} \alpha_{2} \beta_{1} \text {, } \\
& \alpha_{2, t_{2}}=\left(-\frac{1}{2} g_{0}-1\right) \alpha_{2, x x}+\frac{1}{2}\left(g_{0}+1\right) \beta_{2} q_{x}+q \beta_{2, x} \\
& +\frac{1}{2}\left(1+g_{0}\right) q r \alpha_{2}-\alpha_{1} \alpha_{2} \beta_{2} \\
& \beta_{1, t_{2}}=\left(\frac{1}{2} g_{0}+1\right) \beta_{1, x x}+\frac{1}{2}\left(g_{0}+1\right) \alpha_{1} r_{x}+r \alpha_{1, x} \\
& -\frac{1}{2}\left(1+g_{0}\right) q r \beta_{1}+\alpha_{1} \beta_{1} \beta_{2}, \\
& \beta_{2, t_{2}}=\left(1-\frac{1}{2} g_{0}\right) \beta_{2, x x}+\frac{1}{2}\left(g_{0}-1\right) \alpha_{2} r_{x}-r \alpha_{2, x} \\
& +\frac{1}{2}\left(g_{0}-1\right) q r \beta_{2}+\alpha_{2} \beta_{1} \beta_{2} \text {. }
\end{aligned}
$$

In particular, letting $g_{0}=1$ in (26), we have

$$
\begin{gathered}
q_{t_{2}}=-\frac{1}{2} q_{x x}+q^{2} r-2 \alpha_{2, x} \alpha_{1}-2 q \alpha_{2} \beta_{1}, \\
r_{t_{2}}=\frac{1}{2} r_{x x}-q r^{2}+2 \beta_{1, x} \beta_{2}+2 r \alpha_{2} \beta_{1}, \\
\alpha_{1_{t_{2}}}=-\frac{1}{2} \alpha_{1, x x}-q \beta_{1, x}-\alpha_{1} \alpha_{2} \beta_{1}, \\
\alpha_{2_{t_{2}}}=-\frac{3}{2} \alpha_{2, x x}+\beta_{2} q_{x}+q \beta_{2, x}+q r \alpha_{2}-\alpha_{1} \alpha_{2} \beta_{2}, \\
\beta_{1_{t_{2}}}=\frac{3}{2} \beta_{1, x x}+\alpha_{1} r_{x}+r \alpha_{1, x}-q r \beta_{1}+\alpha_{1} \beta_{1} \beta_{2}, \\
\beta_{t_{t_{2}}}=\frac{1}{2} \beta_{2, x x}-r \alpha_{2, x}+\alpha_{2} \beta_{1} \beta_{2},
\end{gathered}
$$


and taking $g_{0}=-1$, (26) becomes

$$
\begin{gathered}
q_{t_{2}}=-\frac{1}{2} q_{x x}+q^{2} r-2 \alpha_{1, x} \alpha_{2}+2 q \alpha_{1} \beta_{2}, \\
r_{t_{2}}=\frac{1}{2} r_{x x}-q r^{2}+2 \beta_{2, x} \beta_{1}-2 r \alpha_{1} \beta_{2}, \\
\alpha_{1_{t_{2}}}=-\frac{3}{2} \alpha_{1, x x}-\beta_{1} q_{x}-q \beta_{1, x}+2 q r \alpha_{1}-\alpha_{1} \alpha_{2} \beta_{1}, \\
\alpha_{2_{t_{2}}}=-\frac{1}{2} \alpha_{2, x x}+q \beta_{2, x}-\alpha_{1} \alpha_{2} \beta_{2}, \\
\beta_{1_{t_{2}}}=\frac{1}{2} \beta_{1, x x}+r \alpha_{1, x}+\alpha_{1} \beta_{1} \beta_{2}, \\
\beta_{2_{t_{2}}}=\frac{3}{2} \beta_{2, x x}-r_{x} \alpha_{2}-r \alpha_{2, x}-q r \beta_{2}+\alpha_{2} \beta_{1} \beta_{2} .
\end{gathered}
$$

If we choose $\alpha_{1}=-\alpha_{2}=\alpha, \beta_{2}=\beta_{1}=\beta$, and $g_{0}=0,(26)$ can be reduced to the second-order super-AKNS equations [10]

$$
\begin{gathered}
q_{t_{2}}=-\frac{1}{2} q_{x x}+q^{2} r+2 q \alpha \beta-2 \alpha \alpha_{x}, \\
r_{t_{2}}=\frac{1}{2} r_{x x}-q r^{2}-2 r \alpha \beta-2 \beta \beta_{x}, \\
\alpha_{t_{2}}=-\alpha_{x x}-q \beta_{x}+\frac{1}{2} q r \alpha-\frac{1}{2} q_{x} \beta, \\
\beta_{t_{2}}=\beta_{x x}+r \alpha_{x}+\frac{1}{2} r_{x} \alpha-\frac{1}{2} q r \beta,
\end{gathered}
$$

which is just the coupled nonlinear Schrödinger equations, also called Manakov equations

$$
\begin{gathered}
u_{T}=-u_{X X}+u^{2} v \\
v_{T}=v_{X X}-u v^{2},
\end{gathered}
$$

as $\alpha=\beta=0, q=u, r=v, t_{2}=T$, and $x=2 X$.

\section{Infinite Conservation Laws}

In what follows, we will derive infinite conservation laws of (25) and (26). From the spectral problem (3), we can introduce the variables

$$
M=\frac{\phi_{2}}{\phi_{1}}, \quad N=\frac{\phi_{3}}{\phi_{1}},
$$

and then we obtain

$$
\begin{gathered}
M_{x}=r+2 \lambda M+\beta_{1} N-q M^{2}-\alpha_{1} M N, \\
N_{x}=\beta_{2}+\alpha_{2} M+\lambda N-q M N .
\end{gathered}
$$

Next, we expand $M$ and $N$ as series of the spectral parameter $\lambda$,

$$
M=\sum_{j=1}^{\infty} M_{j} \lambda^{-j}, \quad N=\sum_{j=1}^{\infty} N_{j} \lambda^{-j}
$$

where $M_{j}$ are even, $p\left(M_{j}\right)=0$, and $N_{j}$ are odd, $p\left(N_{j}\right)=1$.
By substituting (33) into (32) and comparing the coefficients of $\lambda$, we raise the recursion formulas for $M_{j}$ and $N_{j}$,

$$
\begin{aligned}
M_{j+1}= & \frac{1}{2}\left(-r \delta_{n, 0}+M_{j, x}-\beta_{1} N_{j}\right. \\
& \left.+q \sum_{l=1}^{j-1} M_{j} M_{j-l}+\alpha_{1} \sum_{l=1}^{j-1} M_{j} N_{j-l}\right), \\
N_{j+1}= & -\beta_{2} \delta_{n, 0}+N_{j, x}-\alpha_{2} M_{j} \\
& +q \sum_{l=1}^{j-1} M_{j} N_{j-l}, \quad(j \geq 2),
\end{aligned}
$$

We write below the first few terms of $M_{j}$ and $N_{j}$ :

$$
\begin{gathered}
M_{1}=-\frac{1}{2} r, \quad N_{1}=-\beta_{2}, \\
M_{2}=-\frac{1}{4} r_{x}+\beta_{1} \beta_{2}, \quad N_{2}=-\beta_{2, x}+\frac{1}{2} r \alpha_{2}, \\
M_{3}=-\frac{1}{8} r_{x x}+\frac{1}{2} \beta_{1, x} \beta_{2}-\beta_{2, x} \beta_{1}+\frac{1}{4} r \alpha_{2} \beta_{1} \\
+\frac{1}{4} r \alpha_{1} \beta_{2}+\frac{1}{8} q r^{2}, \\
N_{3}=-\beta_{2, x x}+\frac{3}{4} \alpha_{2} r_{x}+\frac{1}{2} r \alpha_{2, x}-\alpha_{2} \beta_{1} \beta_{2}+\frac{1}{2} q r \beta_{2} .
\end{gathered}
$$

On the other hand, it is easy to see that

$$
\frac{\partial}{\partial t} \frac{\partial}{\partial x} \log \phi_{1}=\frac{\partial}{\partial x} \frac{\partial}{\partial t} \log \phi_{1} \quad \text { or } \frac{\partial}{\partial t} \frac{\phi_{1, x}}{\phi_{1}}=\frac{\partial}{\partial x} \frac{\phi_{1, t}}{\phi_{1}}
$$

which implies

$$
\frac{\partial}{\partial t}\left(-\lambda+q M+\alpha_{1} N\right)=\frac{\partial}{\partial x}\left(\frac{1}{2}(A+G)+B M+\rho N\right),
$$

and then the form of the conservation law is

$$
\mu_{t}=v_{x}
$$

with the assumption that $\mu=-\lambda+q M+\alpha_{1} N, \nu=(1 / 2)(A+$ $G)+B M+\rho N$.

With regard to (25), we have

$$
\begin{gathered}
A=-2 \lambda, \quad B=q, \quad G=-g_{0} \lambda, \\
\rho=\left(1-\frac{1}{2} g_{0}\right) \alpha_{1} .
\end{gathered}
$$

Expanding $\mu$ and $\nu$ as

$$
\mu=-\lambda+\sum_{j=1}^{\infty} \mu_{j} \lambda^{-j}, \quad \nu=-\left(1+\frac{1}{2} g_{0}\right) \lambda+\sum_{j=1}^{\infty} v_{j} \lambda^{-j}
$$


then we have

$$
\begin{array}{r}
\mu_{j}=q M_{j}+\alpha_{1} N_{j}, \quad v_{j}=q M_{j}+\left(1-\frac{1}{2} g_{0}\right) \alpha_{1} N_{j}, \\
j \geq 1,
\end{array}
$$

with the coefficients $\mu_{j}$ and $\nu_{j}$, which are called conserved densities and currents, respectively. Then the first two conserved densities and currents of (25) read

$$
\begin{gathered}
\mu_{1}=-\frac{1}{2} q r-\alpha_{1} \beta_{2}, \\
\mu_{2}=-\frac{1}{4} q r_{x}+q \beta_{1} \beta_{2}-\alpha_{1} \beta_{2, x}+\frac{1}{2} r \alpha_{1} \alpha_{2}, \\
\nu_{1}=-\frac{1}{2} q r-\left(1-\frac{1}{2} g_{0}\right) \alpha_{1} \beta_{2}, \\
\nu_{2}=-\frac{1}{4} q r_{x}+q \beta_{1} \beta_{2}-\left(1-\frac{1}{2} g_{0}\right) \alpha_{1} \beta_{2, x} \\
+\frac{1}{2}\left(1-\frac{1}{2} g_{0}\right) r \alpha_{1} \alpha_{2} .
\end{gathered}
$$

So the first two conservation laws of (25) are revealed as

$$
\begin{gathered}
\left(-\frac{1}{2} q r-\alpha_{1} \beta_{2}\right)_{t}=\left[-\frac{1}{2} q r-\left(1-\frac{1}{2} g_{0}\right) \alpha_{1} \beta_{2}\right]_{x}, \\
\left(-\frac{1}{4} q r_{x}+q \beta_{1} \beta_{2}-\alpha_{1} \beta_{2, x}+\frac{1}{2} r \alpha_{1} \alpha_{2}\right)_{t} \\
=\left[-\frac{1}{4} q r_{x}+q \beta_{1} \beta_{2}-\left(1-\frac{1}{2} g_{0}\right) \alpha_{1} \beta_{2, x}\right. \\
\left.+\frac{1}{2}\left(1-\frac{1}{2} g_{0}\right) r \alpha_{1} \alpha_{2}\right]_{x}
\end{gathered}
$$

For (26), one infers

$$
\begin{gathered}
A=-2 \lambda^{2}+q r+\alpha_{1} \beta_{2}-\alpha_{2} \beta_{1}-\frac{1}{2} g_{0} \alpha_{1} \beta_{2}-\frac{1}{2} g_{0} \alpha_{2} \beta_{1}, \\
G=-g_{0} \lambda^{2}+\alpha_{1} \beta_{2}+\alpha_{2} \beta_{1}-\frac{1}{2} g_{0} \alpha_{1} \beta_{2}+\frac{1}{2} g_{0} \alpha_{2} \beta_{1}, \\
\rho=\left(1-\frac{1}{2} g_{0}\right) \lambda \alpha_{1}+\left(\frac{1}{2} g_{0}-1\right) \alpha_{1, x}+\frac{1}{2} g_{0} q \beta_{1}, \\
B=\lambda q-\frac{1}{2} q_{x}+\frac{1}{2} g_{0} \alpha_{1} \alpha_{2} .
\end{gathered}
$$

If we write $\mu$ and $\nu$ as

$$
\mu=-\lambda+\sum_{j=1}^{\infty} \mu_{j} \lambda^{-j}, \quad v=-\left(1+\frac{1}{2} g_{0}\right) \lambda^{2}+\sum_{j=1}^{\infty} v_{j} \lambda^{-j}
$$

the first two are

$$
\begin{gathered}
\mu_{1}=-\frac{1}{2} q r-\alpha_{1} \beta_{2}, \\
\mu_{2}=-\frac{1}{4} q r_{x}+q \beta_{1} \beta_{2}-\alpha_{1} \beta_{2, x}+\frac{1}{2} r \alpha_{1} \alpha_{2}, \\
v_{1}=-\frac{1}{2} g_{0}\left(\beta_{2, x} \alpha_{1}+r \alpha_{1} \alpha_{2}+\alpha_{1, x} \beta_{2}+q \beta_{1} \beta_{2}\right) \\
-\frac{1}{4} q r_{x}+q \beta_{1} \beta_{2}+\frac{1}{4} q_{x} r+\alpha_{1, x} \beta_{2} \\
+\beta_{2, x} \alpha_{1}+\frac{1}{2} r \alpha_{1} \alpha_{2}, \\
+\frac{1}{2} g_{0}\left(\alpha_{1, x} \beta_{2, x}+\frac{1}{2} q r \alpha_{2} \beta_{1}+\frac{1}{2} q r \alpha_{1} \beta_{2}-q \beta_{2, x} \beta_{1}\right. \\
+\frac{1}{2} q \beta_{1, x} \beta_{2}-q \beta_{2, x} \beta_{1}+\frac{1}{8} q_{x} r_{x} \\
+\beta_{2, x x} \alpha_{1}+\frac{1}{4} r_{x} \alpha_{1} \alpha_{2}+\frac{1}{8} q_{1}^{2} \beta_{2}+\frac{3}{4} q r \alpha_{1} \beta_{2} . \\
-\frac{1}{4} q r \alpha_{2} \beta_{1}-\frac{1}{2} r \alpha_{2, x} \alpha_{1} \\
\left.-\frac{1}{2} r \alpha_{1, x} \alpha_{2}-\frac{1}{2} r \alpha_{2, x} \alpha_{1}-\alpha_{1} \alpha_{2} \beta_{1} \beta_{2}+\beta_{2, x x} \alpha_{1}\right)+\alpha_{1, x} \beta_{2, x}
\end{gathered}
$$

The recursion relations for $\mu_{j}$ and $\nu_{j}(j \geq 1)$ are as follows:

$$
\begin{gathered}
\mu_{j}=q M_{j}+\alpha_{1} N_{j}, \\
v_{j}=q M_{j+1}+\frac{1}{2}\left(g_{0} \alpha_{1} \alpha_{2}-q_{x}\right) M_{j}+\left(1-\frac{1}{2} g_{0}\right) \alpha_{1} N_{j+1} \\
-\left(\alpha_{1, x}-\frac{1}{2} g_{0} \alpha_{1, x}-\frac{1}{2} g_{0} q \beta_{2}\right) N_{j},
\end{gathered}
$$

where $M_{j}$ and $N_{j}$ can be recursively calculated from (34). Then we display the first two conservation laws of (26) as

$$
\mu_{1, t}=v_{1, x}, \quad \mu_{2, t}=v_{2, x},
$$

where $\mu_{1}, \mu_{2}, \nu_{1}$, and $\nu_{2}$ are defined in (46).

\section{Concluding Remarks}

In this paper, we proposed a six-component super-AKNS system from a $3 \times 3$ matrix superspectral problem. We obtained the super-Hamiltonian structure and different reductions for the superintegrable equations. Infinitely many conservation laws were also considered. The obtained results supplement the existing theories on the superintegrable systems. The bosonization approach for supersymmetric systems is 
a powerful tool to generate exact solutions. The superintegrable system (23) may admit bosonization. This and other related issues may be considered in further publication.

\section{Conflict of Interests}

The authors declare that there is no conflict of interests regarding the publication of this paper.

\section{Acknowledgments}

This work was supported by Program for Liaoning Excellent Talents in University (Grant no. LJQ2011136), the Natural Science Foundation of China (Grants nos. 11401392, 61372195, and 61304069), and the Key Technologies R \& D Program of Liaoning Province (Grant no. 2011224006).

\section{References}

[1] M. J. Ablowitz, D. J. Kaup, A. C. Newell, and H. Segur, "The inverse scattering transform-Fourier analysis for nonlinear problems," Studies in Applied Mathematics, vol. 53, no. 4, pp. 249-315, 1974.

[2] F. C. You and T. C. Xia, "Integrable couplings of the generalized AKNS hierarchy with an arbitrary function and its bi-Hamiltonian structure," International Journal of Theoretical Physics, vol. 46, no. 12, pp. 3159-3168, 2007.

[3] W. X. Ma, "Integrable couplings and matrix loop algebra," in Nonlinear and Modern Mathematical Physics, W. X. Ma and D. Kaup, Eds., vol. 1562 of AIP Conference Proceedings, pp. 105-122, American Institute of Physics, Melville, NY, USA, 2013.

[4] V. E. Zakharov and A. B. Shabat, "Interaction between solitons in a stable medium," Soviet Physics-JETP, vol. 34, pp. 62-69, 1973.

[5] B. A. Kupershmidt, "A super Korteweg-de Vries equation: an integrable system," Physics Letters A, vol. 102, no. 5-6, pp. 213$215,1984$.

[6] M. Gürses and Ö. Oğuz, "A super AKNS scheme," Physics Letters A, vol. 108, no. 9, pp. 437-440, 1985.

[7] M. Gürses and Ö. Oğuz, "A super soliton connection," Letters in Mathematical Physics, vol. 11, no. 3, pp. 235-246, 1986.

[8] Y. S. Li and L. N. Zhang, "Hamiltonian structure of the super evolution equation," Journal of Mathematical Physics, vol. 31, no. 2, pp. 470-475, 1990.

[9] W. Ma, J. He, and Z. Qin, "A supertrace identity and its applications to superintegrable systems," Journal of Mathematical Physics, vol. 49, no. 3, Article ID 033511, 2008.

[10] F. C. You, "Nonlinear super integrable Hamiltonian couplings," Journal of Mathematical Physics, vol. 52, no. 12, Article ID 123510, 2011.

[11] F. C. You, "Nonlinear super integrable couplings of super Dirac hierarchy and its super Hamiltonian structures," Communications in Theoretical Physics, vol. 57, no. 6, pp. 961-966, 2012.

[12] J. Zhang, F. C. You, and Y. Zhao, "A new super extension of Dirac hierarchy," Abstract and Applied Analysis, vol. 2014, Article ID 472101, 6 pages, 2014.

[13] Q. P. Liu, "Darboux transformations for supersymmetric Korteweg-de Vries equations," Letters in Mathematical Physics, vol. 35, no. 2, pp. 115-122, 1995.
[14] X. B. Hu, "A powerful approach to generate new integrable systems," Journal of Physics A: Mathematical and General, vol. 27, no. 7, pp. 2497-2514, 1994.

[15] M. Gurses and O. Oguz, "A super soliton connection," Letters in Mathematical Physics, vol. 11, no. 3, pp. 235-246, 1986.

[16] M. Gurses, S. Salihoglu, and O. Oguz, "Integrable nonlinear partial differential equations on homogenous spaces," International Journal of Modern Physics A, vol. 5, no. 9, pp. 1801-1817, 1990.

[17] X. B. Hu, "An approach to generate superextensions of integrable systems," Journal of Physics A: Mathematical and General, vol. 30, no. 2, pp. 619-632, 1997. 


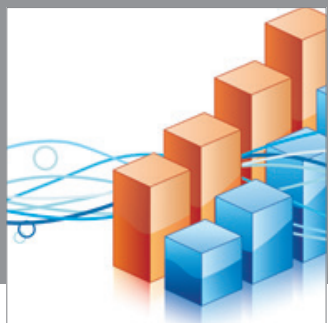

Advances in

Operations Research

mansans

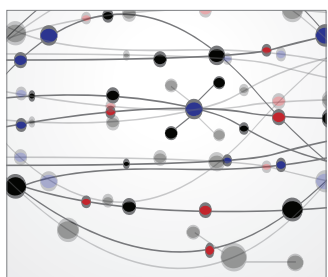

The Scientific World Journal
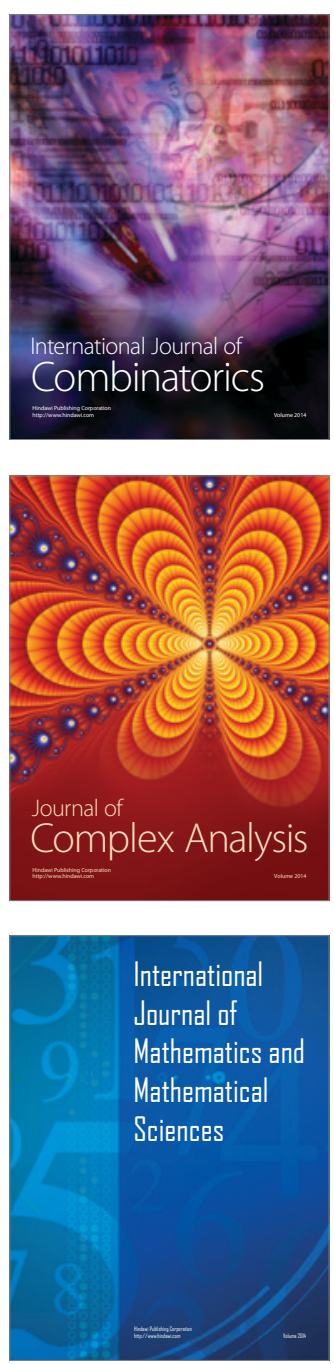
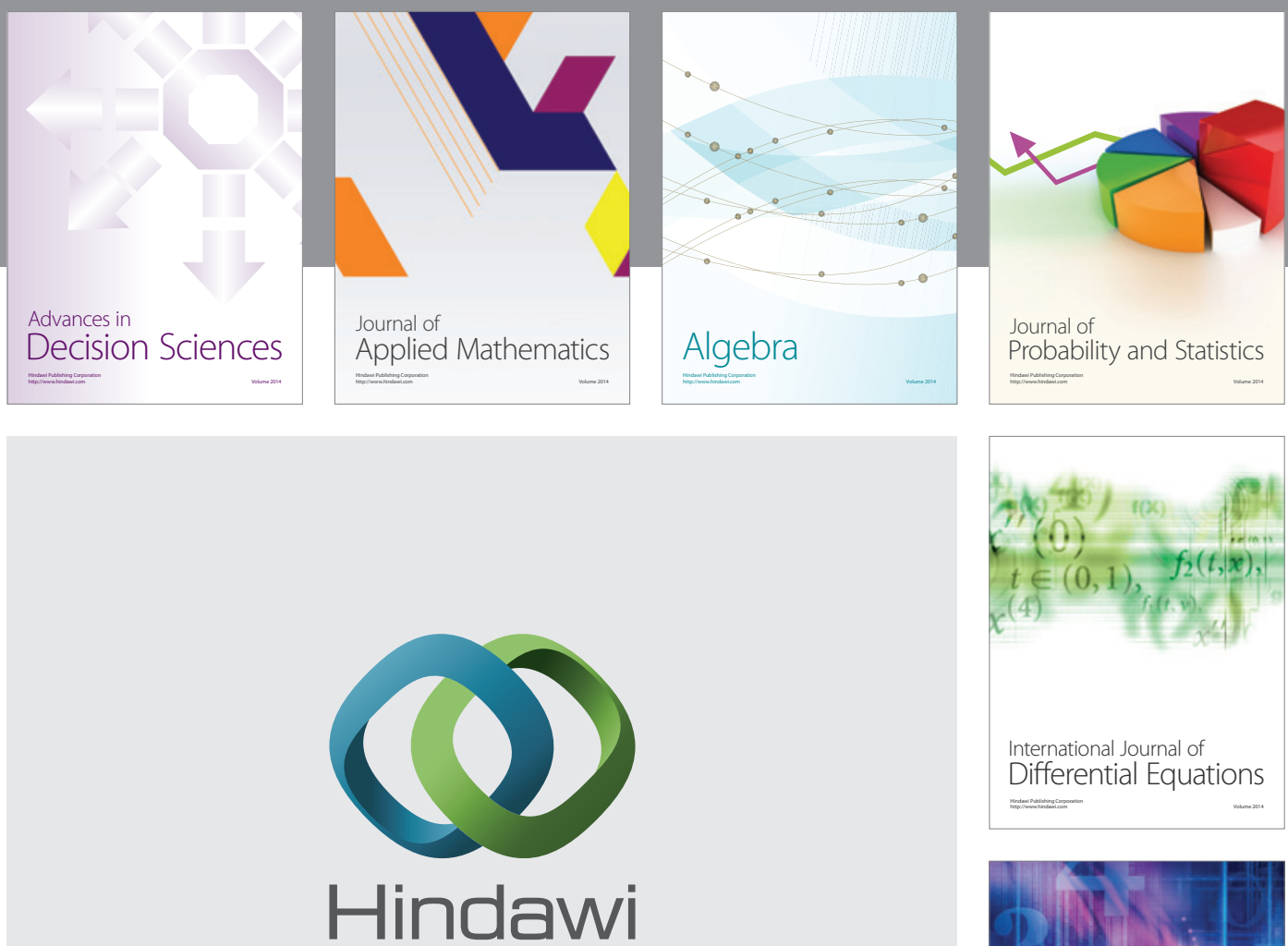

Submit your manuscripts at http://www.hindawi.com
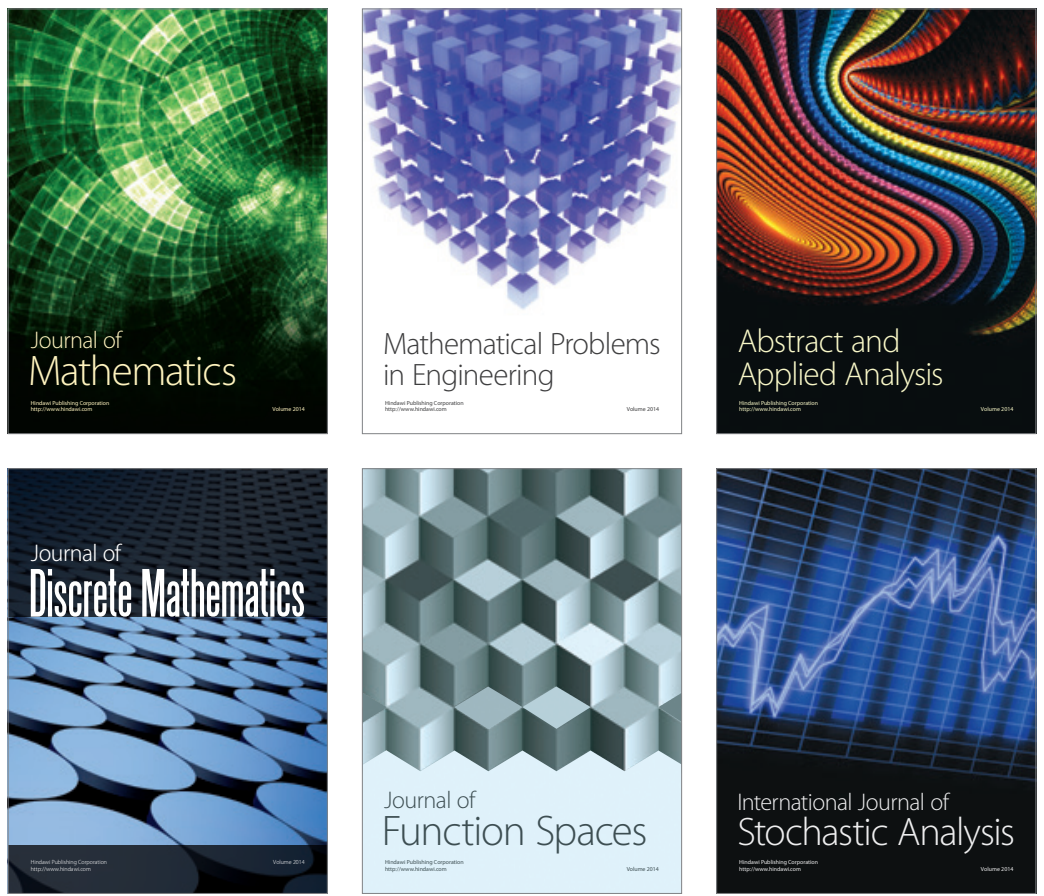

Journal of

Function Spaces

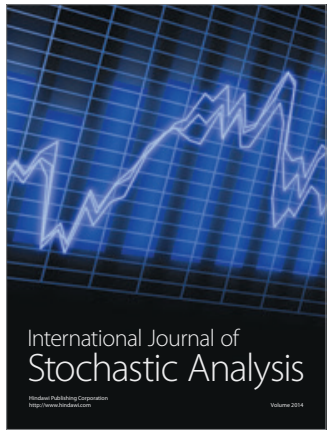

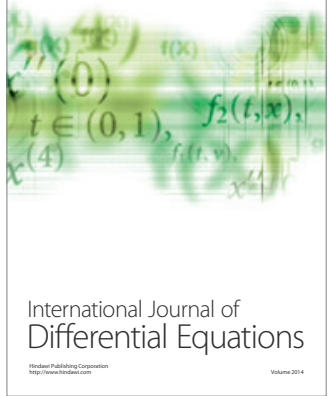
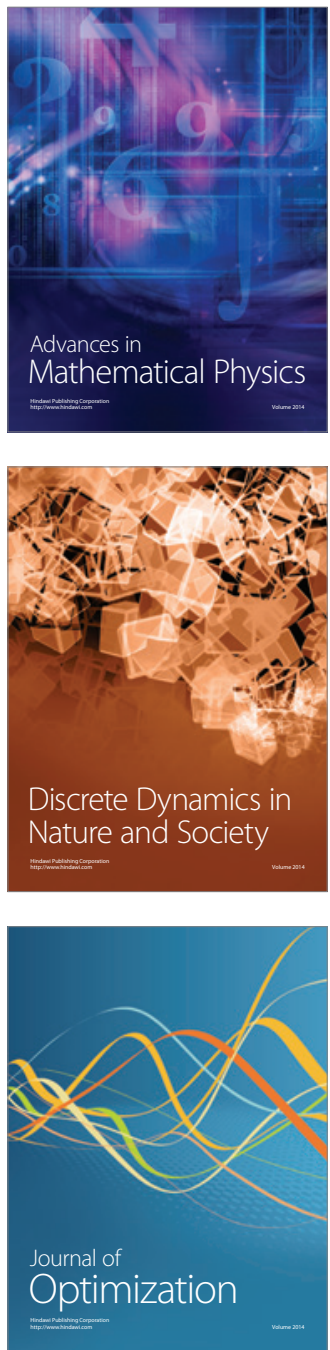